\title{
The theory of frequency shifts as a function of the spin location, using the oscillating cantilever-driven adiabatic reversals technique
}

\author{
G.P. Berman, ${ }^{1}$ F. Borgonovi, ${ }^{2,3}$ and V.I. Tsifrinovich ${ }^{4}$ \\ ${ }^{1}$ Theoretical Division and CNLS, MS B213, Los Alamos National Laboratory, Los Alamos, NM 87545 \\ ${ }^{2}$ Dipartimento di Matematica e Fisica, Universitá Cattolica, via Musei 41, 25121 Brescia, Italy \\ ${ }^{3}$ INFN, Sezione di Pavia, Italy \\ ${ }^{4}$ IDS Department, Polytechnic University, Six Metrotech Center, Brooklyn, New York 11201
}

(Dated: April 25, 2018)

\begin{abstract}
The theory of the oscillating cantilever-driven adiabatic reversals (OSCAR) in magnetic resonance force microscopy (MRFM) is extended to describe the relation between an external magnetic field and a dipole magnetic field for an arbitrary location of the single spin. An analytical estimate for the OSCAR MRFM frequency shift is derived and shown to be in excellent agreement with numerical simulations. The dependence of the frequency shift on the position of the spin relative to the cantilever has characteristic maxima and minima which can be used to determine the spin location experimentally.
\end{abstract}

PACS numbers: 76.60.-k, 07.55.-w

\section{INTRODUCTION}

The oscillating cantilever driven adiabatic reversals (OSCAR) technique in magnetic resonance force microscopy (MRFM) introduced in [1] has been used to successfully detect a single electron spin below the surface of a solid [2]. In the OSCAR MRFM technique the vibrations of the cantilever tip (CT) with an attached ferromagnetic particle in presence of a $r f$ magnetic field cause the periodic reversals of the effective magnetic field acting on the single electron spin. If the conditions of adiabatic motion are satisfied the spin follows the effective magnetic field. The back action of the spin on the CT causes a small frequency shift of the $\mathrm{CT}$ vibrations, which can be measured with high precision.

The quasiclassical theory of OSCAR MRFM has been developed in [3]. This theory contains two important limitations. First, it assumes that the external magnetic field $\vec{B}_{e x t}$ at the spin is much greater than the dipole field $\vec{B}_{d}$ produced by the ferromagnetic particle. In real experiments, in order to increase the frequency shift $\delta \omega_{c}$, one has to decrease the distance between the $\mathrm{CT}$ and the spin to values where the dipole field becomes sometimes greater than the external field [2]. Second, it was assumed in [3] that the spin is located in the plane of the cantilever vibrations. Thus, the quasiclassical theory should be extended in order to describe both an arbitrary relation between $\vec{B}_{\text {ext }}$ and $\vec{B}_{d}$ and an arbitrary location of the spin. This extension is presented in our paper.

A single spin is a quantum object which must be described using quantum theory. The quantum theory of OSCAR MRFM has been developed in [4] with the same limitations as the quasiclassical theory. It was found, as may be expected, that the frequency shift $\delta \omega_{c}$ in quantum theory may assume only two values $\pm\left|\delta \omega_{c}\right|$ corresponding to the two directions of the spin relative to the effective magnetic field. The value of $\left|\delta \omega_{c}\right|$ in quantum theory is the same as the maximum frequency shift calculated

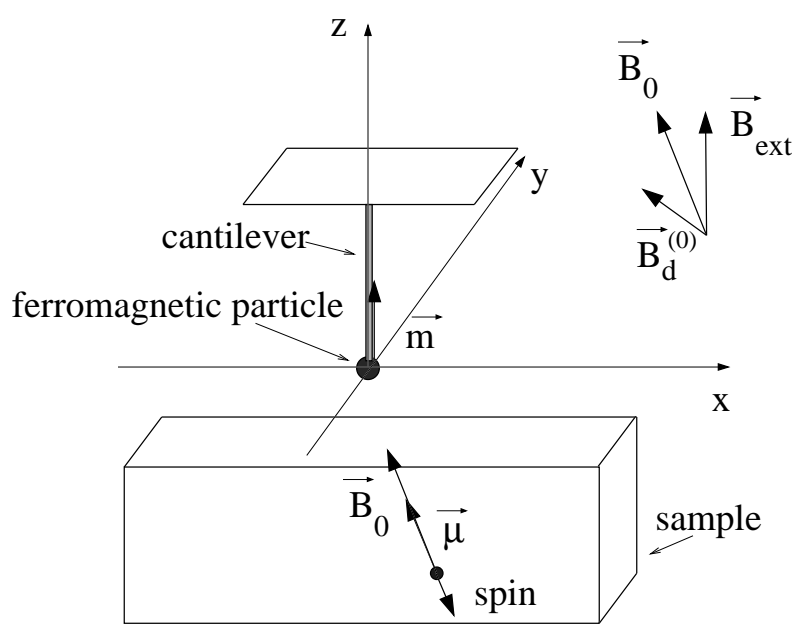

FIG. 1: MRFM setup. The equilibrium position of the spin and the cantilever with a spherical ferromagnetic particle. $\vec{m}$ is the magnetic moment of the ferromagnetic particle, $\vec{\mu}$ is the magnetic moment of the spin, $\vec{B}_{e x t}, \vec{B}_{d}^{(0)}$ and $\vec{B}_{0}$ are respectively the external permanent magnetic field, the dipole field on the spin, and the net magnetic field. In general the vectors $\vec{B}_{d}^{(0)}$ and $\vec{B}_{0}$ do not lie in the $x-z$ plane.

using quasiclassical theory (where it can take any value between $-\left|\delta \omega_{c}\right|$ and $\left.\left|\delta \omega_{c}\right|\right)$. Thus, to calculate the quantum frequency shift, it is reasonable to use quasiclassical instead of quantum theory.

\section{EQUATIONS OF MOTION}

We consider the MRFM setup shown in Fig. [1 The CT oscillates in the $x-z$ plane. The origin is placed at the equilibrium position of the center of the ferromagnetic particle). Note that here we ignore the static displacement of the CT caused by the magnetic force of the spin. The magnetic moment of the spin, $\vec{\mu}$ shown in 
Fig. 11 points initially in the direction of the magnetic field $\vec{B}_{0}$, which corresponds to the equilibrium position of CT. (See Eq. (4)). We assume now that the $r f$ magnetic field $2 \vec{B}_{1}$ is linearly polarized in the plane which is perpendicular to $\vec{B}_{0}$. (Later we will generalize this to an arbitrary direction of polarization). The dipole magnetic field $\vec{B}_{d}$ is given by:

$$
\vec{B}_{d}=\frac{\mu_{0}}{4 \pi} \frac{3(\vec{m} \cdot \vec{n}) \vec{n}-\vec{m}}{r_{v}^{3}}
$$

where $\vec{m}$ is the magnetic moment of the ferromagnetic particle pointing in the positive $z$-direction, $r_{v}$ is the (variable) distance between the moving $\mathrm{CT}$ and the stationary spin and $\vec{n}$ is the unit vector pointing from the CT to the spin. We define:

$$
\begin{aligned}
r_{v} & =\sqrt{\left(x-x_{c}\right)^{2}+y^{2}+z^{2}}, \\
\vec{n} & =\left(\frac{x-x_{c}}{r_{v}}, \frac{y}{r_{v}}, \frac{z}{r_{v}}\right),
\end{aligned}
$$

where $x, y, z$ are the spin coordinates, and $x_{c}$ is the CTcoordinate (i.e. the coordinate of the center of the ferromagnetic particle). At the equilibrium, the net magnetic field at the spin is,

$$
\begin{gathered}
\vec{B}_{0}=\vec{B}_{e x t}+\vec{B}_{d}^{(0)} \\
\vec{B}_{d}^{(0)}=\frac{3 m \mu_{0}}{4 \pi r^{5}}\left(z x, z y, z^{2}-\frac{r^{2}}{3}\right), \\
\vec{B}_{e x t}=\left(0,0, B_{e x t}\right),
\end{gathered}
$$

where $r=\sqrt{x^{2}+y^{2}+z^{2}}$. In the approximation which is linear in $x_{c}$, the magnetic field $\vec{B}_{d}$ changes by the value of $\vec{B}_{d}^{(1)}$ :

$$
\begin{gathered}
\vec{B}_{d}^{(1)}=-\left(G_{x}, G_{y}, G_{z}\right) x_{c} \\
\left(G_{x}, G_{y}, G_{z}\right)=\frac{3 m \mu_{0}}{4 \pi r^{7}}\left(z\left(r^{2}-5 x^{2}\right),-5 x y z, x\left(r^{2}-5 z^{2}\right)\right),
\end{gathered}
$$

where $\left(G_{x}, G_{y}, G_{z}\right)$ describes the gradient of the magnetic field at the spin location at $x_{c}=0$ :

$$
\left(G_{x}, G_{y}, G_{z}\right)=\left(\frac{\partial B_{d}^{x}}{\partial x}, \frac{\partial B_{d}^{y}}{\partial x}, \frac{\partial B_{d}^{z}}{\partial x}\right) .
$$

(Note that the magnetic field and its gradient depend on the CT coordinate $x_{c}$ ). Next we consider the equation of motion for the spin magnetic moment $\vec{\mu}$ in the system of coordinates rotating with the $r f$ field at frequency $\omega$ about the magnetic field $\vec{B}_{0}$. (The $\tilde{z}$ axis of this new system points in the direction of $\left.\vec{B}_{0}\right)$. We have:

$$
\begin{aligned}
\overrightarrow{\dot{\mu}} & =-\gamma \vec{\mu} \times \vec{B}_{e f f}, \\
\vec{B}_{e f f} & =\left(B_{1}, 0, B_{0}-(\omega / \gamma)-x_{c} \sum_{i} G_{i} \cos \alpha_{i}\right), \\
\cos \alpha_{i} & =B_{0}^{i} / B_{0} .
\end{aligned}
$$

Here $\alpha_{i},(i=x, y, z)$ are the angles between the direction of the magnetic field $\vec{B}_{0}$ and the axes $x, y, z$ of the laboratory system of coordinates; and $\gamma$ is the gyromagnetic ratio of the electron spin. ( $\gamma$ is the absolute value of the gyromagnetic ratio). We ignore the transverse components of the dipole field $\vec{B}_{d}$ because they represent the fast oscillating terms in the rotating system of coordinate. Also we consider only the rotating component of the $r f$ magnetic field.

The equations of motion for the CT can be written,

$$
\ddot{x}_{c}+\omega_{c}^{2} x_{c}=F_{x} / m^{*},
$$

where $\omega_{c}$ and $m^{*}$ are the frequency and the effective mass of the $\mathrm{CT}$ and $F_{x}$ is the magnetic force acting on the ferromagnetic particle on CT. We consider the CT oscillations in the laboratory system of coordinates. Ignoring fast oscillating terms in the laboratory system, we obtain:

$$
F_{x}=-\mu_{\tilde{z}} \sum_{i} G_{i} \cos \alpha_{i}
$$

Next, we will use the following units: for time $1 / \omega_{c}$, for magnetic moment $\mu_{B}$, for magnetic field $\omega_{c} / \gamma$, for length the characteristic distance $L_{0}$ between $\mathrm{CT}$ and the spin, for force $k_{c} L_{0}$, where $k_{c}=m^{*} \omega_{c}^{2}$ is the effective CT spring constant. Using these units, we derive the following dimensionless equations of motion:

$$
\begin{aligned}
& \dot{\vec{\mu}}=-\vec{\mu} \times \vec{B}_{e f f}, \\
& \ddot{x}_{c}+x_{c}=F_{x}, \\
& \vec{B}_{e f f}=\left(B_{1}, 0, \Delta-\beta \mathcal{G} x_{c}\right), \\
& F_{x}=-\alpha \beta \mathcal{G} \mu_{\tilde{z}}, \\
& \Delta=B_{0}-\omega \\
& \mathcal{G}=\frac{1}{r^{7}}\left[z\left(r^{2}-5 x^{2}\right) \cos \alpha_{x}-5 x y z \cos \alpha_{y}\right. \\
& \left.+x\left(r^{2}-5 z^{2}\right) \cos \alpha_{z}\right] .
\end{aligned}
$$

The parameters $\alpha$ and $\beta$ are given by: 


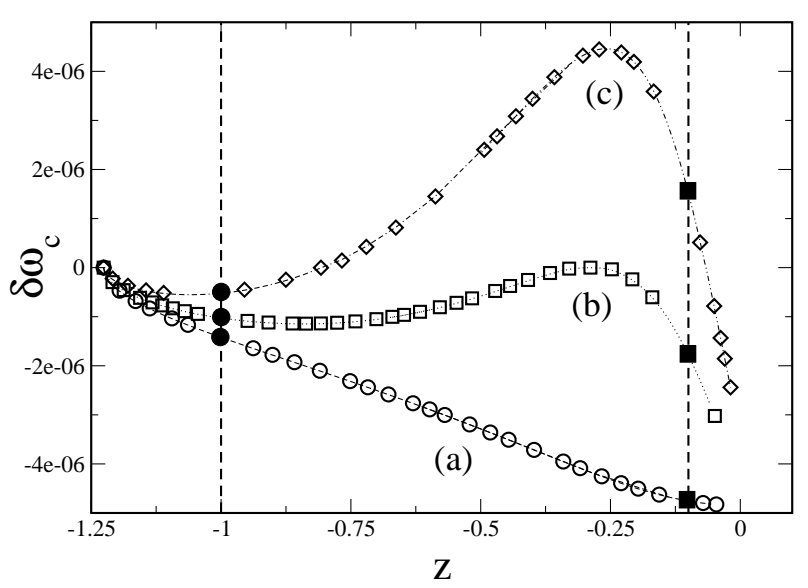

FIG. 2: The OSCAR MRFM frequency shift $\delta \omega_{c}(z)$ at the central resonant surface $(\Delta=0)$, for $x>0$. The symbols show the numerical data, the lines correspond to the estimate (17) for (a) $y=0$ (circles), (b) $y=x / 2$ (squares) and (c) $y=x$ (diamonds). Solid squares and circles indicate frequency shifts at the spin locations indicated in Fig. [3] In all Figures the coordinates $x, y$ and $z$ are in units of $L_{0}$ and the frequencies are in units of $\omega_{c}$.

$$
\alpha=\frac{\mu_{B} \omega_{c}}{\gamma k_{c} L_{0}^{2}}, \quad \beta=\frac{3 \gamma \mu_{0} m}{4 \pi \omega_{c} L_{0}^{3}} .
$$

Note that all quantities in Eq. (13) are dimensionless, i.e. $x$ means $x / L_{0}, \mu$ means $\mu / \mu_{B}, B_{0}$ means $\gamma B_{0} / \omega_{c}$ and so on. In terms of dimensional quantities the parameter $\beta$ is the ratio of the dipole frequency $\gamma B_{d}^{(0)}$ to the CT frequency $\omega_{c}$, and the product $\alpha \beta$ is the ratio of the static CT displacement $F_{x} / k_{c}$ to the CT-spin distance $L_{0}$. The derived equations are convenient for both numerical simulations and analytical estimates.

\section{THE OSCAR MRFM FREQUENCY SHIFT}

In this section we present the analytical estimates and the numerical simulations for the OSCAR MRFM frequency shift. When the CT oscillates, the resonant condition $\omega=\gamma\left|\vec{B}_{\text {ext }}+\vec{B}_{d}\right|$ can be satisfied only if the spin is located inside the resonant slice which is defined by its boundaries:

$$
\left|\vec{B}_{\text {ext }}+\vec{B}_{d}\left(x_{c}= \pm A\right)\right|=\omega / \gamma
$$

where $A$ is the amplitude of the CT vibrations. For an analytical estimate, we assume that the spin is located at the central surface of the resonant slice. In this case in Eq. (13) $\Delta=0$.

To obtain an analytical estimate for the OSCAR MRFM frequency shift we will assume an ideal adiabatic motion and put $\overrightarrow{\dot{\mu}}=0$ in Eq. (13). Let the CT begin its motion (at $t=0)$ from the right end position $x_{c}(0)=A$.
Then the initial direction (i.e. at $t=0$ ) of the effective magnetic field $\vec{B}_{e f f}$ relative to the magnetic field $\vec{B}_{e x t}+\vec{B}_{d}$ and of the magnetic moment $\vec{\mu}$ depends on the sign of $\mathcal{G}: \vec{B}_{\text {eff }}$ and $\vec{\mu}$ have the same direction for $\mathcal{G}<0$ and opposite directions for $\mathcal{G}>0$. Substituting the derived expression for $\mu_{\tilde{z}} \simeq-B_{\text {eff }}^{z} \mathcal{G} /\left|\overrightarrow{B_{\text {eff }}}\right||\mathcal{G}|$ into $F_{x}$ we obtain the following equation for $x_{c}$ :

$$
\ddot{x}_{c}+x_{c}\left\{1+\frac{\alpha \beta^{2} \mathcal{G}|\mathcal{G}|}{\sqrt{B_{1}^{2}+\left(\beta \mathcal{G} x_{c}\right)^{2}}}\right\}=0 .
$$

We solve this equation as in 3 , using the perturbation theory of Bogoliubov and Mitropolsky [5], and we find the dimensionless frequency shift (see Appendix):

$$
\begin{aligned}
& \delta \omega_{c} \simeq \frac{2}{\pi} \frac{\alpha \beta^{2} \mathcal{G}|\mathcal{G}|}{\sqrt{B_{1}^{2}+(\beta \mathcal{G} A)^{2}}}\{1+ \\
& \left.\frac{1}{2} \frac{B_{1}^{2}}{B_{1}^{2}+(\beta \mathcal{G A})^{2}}\left[\ln \left(\frac{4 \sqrt{B_{1}^{2}+(\beta \mathcal{G A})^{2}}}{B_{1}}\right)+\frac{1}{2}\right]\right\}
\end{aligned}
$$

In typical experimental conditions we have

$$
B_{1} \ll \beta \mathcal{G} A,
$$

and Eq. (17) transforms to the simple expression

$$
\delta \omega_{c}=\frac{2}{\pi} \frac{\alpha \beta \mathcal{G}}{A} .
$$

One can see that the frequency shift is determined by the ratio of the static $\mathrm{CT}$ displacement $F_{x} / k_{c}$ to the amplitude of the $\mathrm{CT}$ vibrations A. We will also present Eq. (17) in terms of dimensionless quantities:

$$
\frac{\delta \omega_{c}}{\omega_{c}}=\frac{2 \mu_{B} G_{0}}{\pi A k_{c}}
$$

where

$$
G_{0}=\sum_{i} G_{i} \cos \alpha_{i}
$$

Eqs. (17) and (19) represent an extension of the estimate derived in [3]. These equations are valid for any point on the central resonant surface and for any relation between $\vec{B}_{\text {ext }}$ and $\vec{B}_{d}$. It follows from Eq. (17) that $\delta \omega_{c}$ is an even function of $y$ and an odd function of $x$.

In our computer simulations we have used the following parameters taken from experiments [2]:

$$
\begin{gathered}
\omega / 2 \pi=5.5 \mathrm{kHz}, \quad k_{c}=110 \mu \mathrm{N} / \mathrm{m}, \quad A=16 \mathrm{~nm}, \\
B_{\text {ext }}=30 \mathrm{mT}, \quad \omega / 2 \pi=2.96 \mathrm{GHz}, \quad \omega / \gamma=106 \mathrm{mT}, \\
\left|G_{z}\right|=2 \times 10^{5} \mathrm{~T} / \mathrm{m}, \quad B_{1}=300 \mu \mathrm{T}, \quad L_{0} \approx 350 \mathrm{~nm} .
\end{gathered}
$$




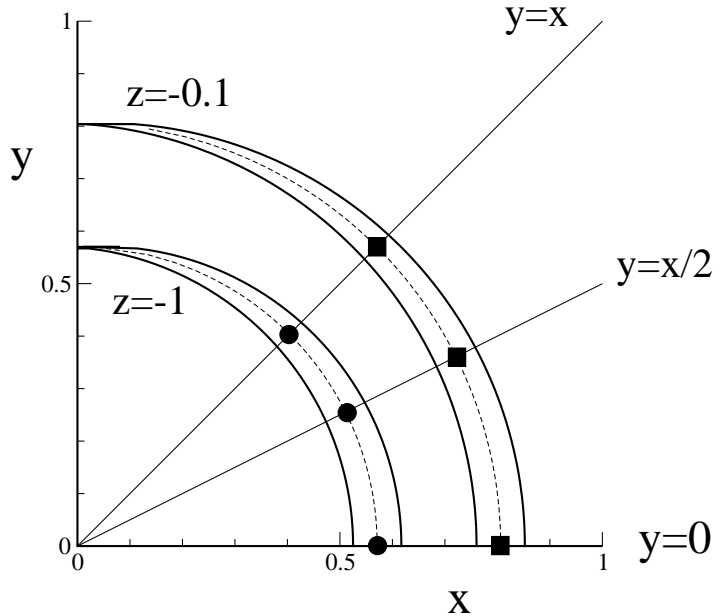

FIG. 3: Cross-sections of the resonant slice for $z=-0.1$ and $z=-1$. The dashed lines show the intersection between the cross sections and the central resonant surface. The solid squares and circles indicate spin locations which correspond to the frequency shifts given by the same symbols in Fig. 2

The corresponding dimensionless parameters are the following:

$$
\begin{gathered}
\alpha=1.35 \times 10^{-13}, \quad \beta=1.07 \times 10^{6}, \quad A=4.6 \times 10^{-2}, \\
B_{1}=1.5 \times 10^{3}, \quad B_{\text {ext }}=1.53 \times 10^{5}, \quad \omega=5.4 \times 10^{5} .
\end{gathered}
$$

As initial conditions we take:

$$
\vec{\mu}(0)=(0,0,1), \quad x_{c}(0)=A, \quad \dot{x}_{c}(0)=0 .
$$

Below we describe the results of our computer simulations. Fig. 2 shows the frequency shift $\delta \omega_{c}$ as a function of the spin $z$-coordinate at the central resonant surface $(\Delta=0)$. First, one can see an excellent agreement between the numerical data and the analytical estimate (17). Second, as expected, the maximum magnitude of the frequency shift $\left|\delta \omega_{c}\right|$ can be achieved when the spin is located in the plane of the CT vibrations $y=0$. However, for $y=x$, it has almost the same magnitude $\left|\delta \omega_{c}\right|$ (with the opposite sign of $\delta \omega_{c}$ ). Moreover, for $y=x$ the dependence $\delta \omega_{c}(z)$ has an extremum, which can be used for the measurement of the spin $z$-coordinate. If the distance between the $\mathrm{CT}$ and the surface of the sample can be controlled, then the "depth" of the spin location below the sample surface can be determined. (In all Figures, the coordinates $x, y$ and $z$ are given in units of $L_{0}$, and the frequency shift is in units of $\omega_{c}$.)

Fig. 3 shows the cross-sections of the resonant slice for $z=-0.1$ and $z=-1$. The greater the distance from the CT, the smaller the cross-sectional area. The solid squares and circles in Fig. 33 show the spin locations which correspond to the frequency shifts indicated by the same symbols in Fig. 2

Fig. 4 demonstrates the "radial" dependence of the frequency shift $\delta \omega_{c}\left(r_{p}\right)$, where $r_{p}=\left(x^{2}+y^{2}\right)^{1 / 2}$. The value a)

b)

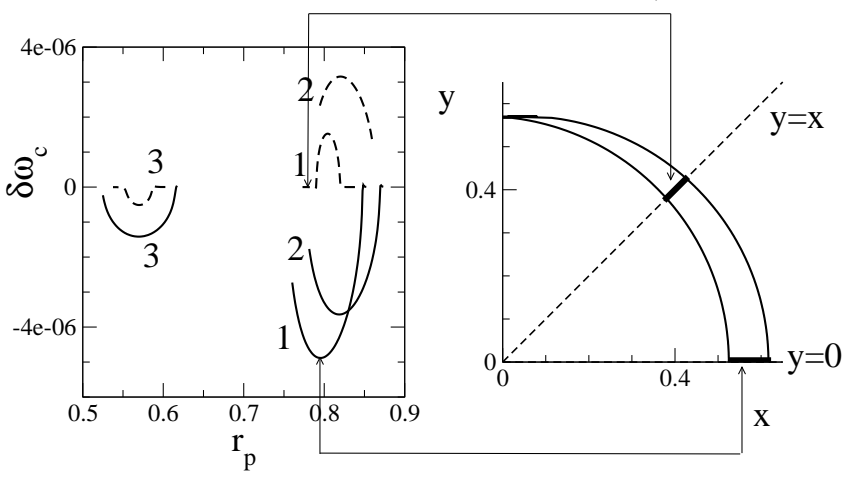

FIG. 4: a) The OSCAR MRFM frequency shift $\delta \omega_{c}\left(r_{p}\right)$ inside the cross-sectional area of the resonant slice for $x>0$. The solid lines correspond to $y=0$ and the dashed lines correspond to $y=x$. Lines are $1-, z=-0.1,2-, z=-0.43$, and $3-, z=-1$. $r_{p}=\left(x^{2}+y^{2}\right)^{1 / 2}$. b) the cross-section of the resonant slice $z=-0.1$. The bold segments show the spin locations which correspond to the lines 1 on $a$ ).

of $r_{p}$ can be changed by the lateral displacement of the cantilever. As one may expect, the maximum value of $\left|\delta \omega_{c}\right|$ corresponds to the central resonant surface. The maximum becomes sharper as z decreases. Thus, a small distance between the $\mathrm{CT}$ and the sample surface is preferable for the measurement of the radial position of the spin.

Fig, 5 shows the "azimuthal dependence" of the frequency shift $\delta \omega_{c}(\phi)$, where $\phi=\tan ^{-1}(y / x)$ and the spin is located on the central resonant surface. Note that for the given values of $z$ and $\phi$, the coordinates $x$ and $y$ of the spin are fixed if the spin is located on the central resonant surface. The value of $\phi$ can be changed by rotating the cantilever about its axis. One can see the sharp extrema of the function $\delta \omega_{c}(\phi)$. Again, the small distance between the $\mathrm{CT}$ and the sample is preferable for the measurement of the "azimuthal position" of the spin.

Finally, we consider the realistic case in which the direction of polarization of the $r f$ field $2 \vec{B}_{1}$ is fixed in the laboratory system of coordinates. Now the angle $\theta$ between the direction of polarization of $2 \vec{B}_{1}$ and the field $\vec{B}_{0}$ depends on the spin coordinate because the magnitude and the direction of the dipole field $\vec{B}_{d}^{(0)}$ depend on the spin location. To describe this case we ignore the component of $2 \vec{B}_{1}$ which is parallel to $\vec{B}_{0}$, and change $B_{1}$ to $B_{1} \sin \theta$ in all our formulas. As an example, Fig. [6 demonstrates the dependence $\delta \omega_{c}(z)$ for the case in which the $r f$ field is polarized along the $x$-axis. One can see that in a narrow region of $z$ the magnitude of the frequency shift sharply drops. This occurs because in this region the magnetic field $\vec{B}_{0}$ is almost parallel to the $x$ axis. Thus, the effective field $B_{1} \sin \theta$ is small: the condition of the adiabatic motion $\gamma\left[B_{1} \sin \theta\right]^{2} \gg\left|d \vec{B}_{\text {eff }} / d t\right|$ is not satisfied; and the spin does not follow the effective magnetic field. The dashed lines in Fig. [6] corre- 
a)

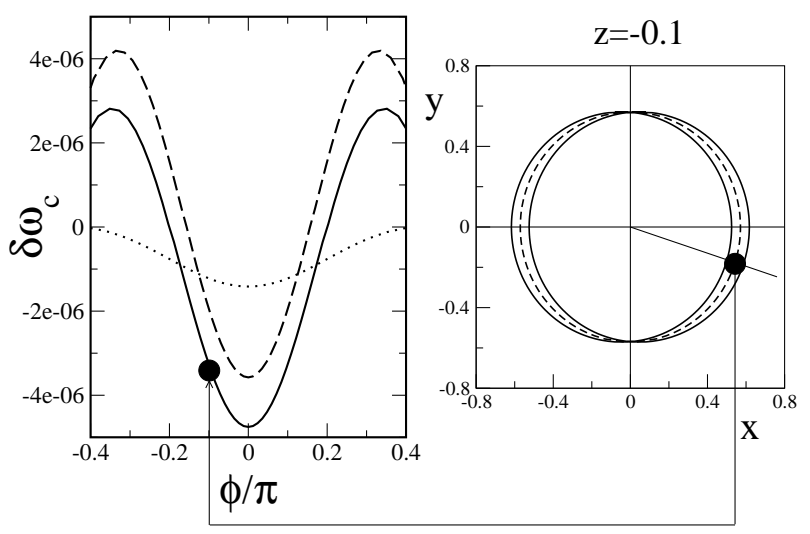

FIG. 5: a) $\delta \omega_{c}(\phi)$, with $\phi=\tan ^{-1}(y / x)$ for the central resonant surface and $z=-0.1$ (full line); $z=-0.43$ (dashed line), $z=-1$ (dotted line). b) solid line shows the crosssection of the resonant slice for $z=-0.1$. Dashed line shows the intersection between the plane $z=-0.1$ and the central resonant surface. The solid circle in b) shows the spin location $\phi / \pi=-0.1$ whose corresponding frequency shift is marked by a solid circle on a).

spond to the analytical estimate (17) with the substitution $B_{1} \rightarrow B_{1} \sin \theta$ : the analytical estimate assumes adiabatic conditions, which are violated for small $\theta$.

The sharp drop of $\left|\delta \omega_{c}\right|$ could be observed either by the change of the distance between the CT and the sample surface or by the change of the direction of polarization of the $r f$ field.

In any case this effect provides an independent measurement of the spin "depth" below the sample surface.

\section{CONCLUSION}

We have derived the quasiclassical equations of motion describing the OSCAR technique in MRFM for arbitrary relation between the external and dipole magnetic fields and arbitrary location of a single spin. We have obtained an analytical estimate of the OSCAR MRFM frequency shift $\delta \omega_{c}$ which is in excellent agreement with numerical simulations. We have shown that the dependence $\delta \omega_{c}$ on the position of spin relative to the cantilever contains characteristic maxima and minima which can be used to determine the position of the spin. We believe that moving cantilever in three dimensions, rotating it (or the sample) about the cantilever's axis and changing the direction of the polarization of the $r f$ magnetic field, experimentalist eventually will enable the determination of the position of a single spin. We hope that our work will help to achieve this goal.
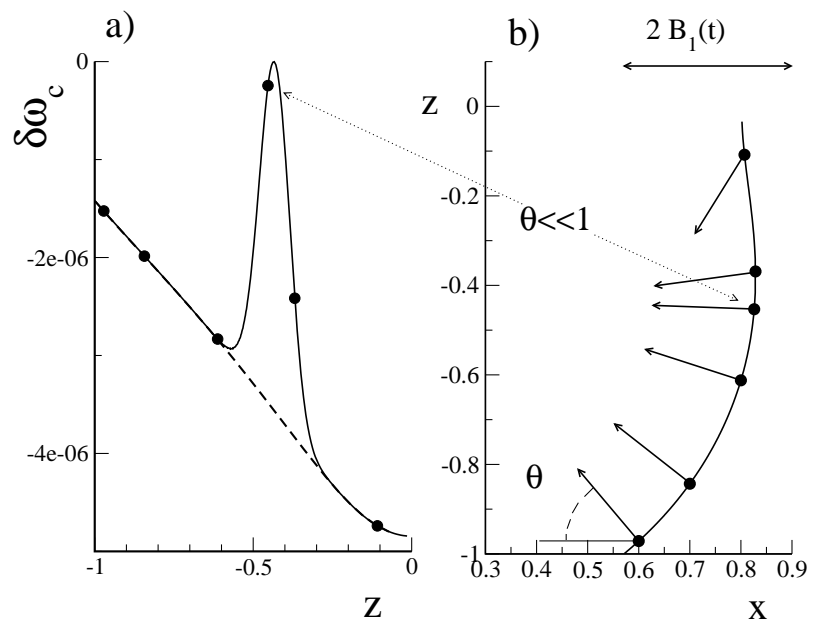

FIG. 6 : a) $\delta \omega_{c}(z)$ when the $r f$ field $\vec{B}_{1}$ is parallel to the $x$-axis. The spin is located at the central resonant surface $y=0, x>$ 0 . Solid line are numerical data, dashed line is the analytical estimate (17), which assumes adiabatic motion of the spin magnetic moment $\vec{\mu}$ parallel to $\vec{B}_{e f f}$. For a few numerical points indicated as solid cirlces in a) the corresponding $\vec{B}_{0}$ field is shown in b). b) solid line : intersection between the central resonant surface and the $x-z$ plane. Arrows show the magnetic field $\vec{B}_{0}$ on this intersection at the points indicated as solid circles in a). The absolute value of the frequency shift $\left|\delta \omega_{c}\right|$ drops at the spin locations where $\vec{B}_{0}$ is approximately parallel to $\vec{B}_{1}(\theta \ll 1)$.

\section{Acknowledgments}

This work was supported by the Department of Energy (DOE) under Contract No. W-7405-ENG-36, by the Defense Advanced Research Projects Agency (DARPA), by the National Security Agency (NSA), and by the Advanced Research and Development Activity (ARDA).

\section{APPENDIX}

Eq. (16) can be written in the following form:

$$
\frac{d^{2} x_{c}}{d \tau^{2}}+x_{c}=\epsilon f\left(x_{c}\right),
$$

where $\tau=\omega_{c} t$ is the dimensionless time,

$$
f\left(x_{c}\right)=\frac{\beta \mathcal{G} x_{c}}{\sqrt{B_{1}^{2}+(\beta \mathcal{G})^{2} x_{c}^{2}}},
$$

and $\epsilon=-\alpha \beta|\mathcal{G}|$. [5]:

The approximate solution of (21), can be written as

$$
x_{c}(\tau)=a(\tau) \cos \psi(\tau)+O(\epsilon) \text {, }
$$


where in the first order in $\epsilon, a(\tau)$ and $\psi(\tau)$ satisfy the equations:

$$
\begin{aligned}
& \frac{d a}{d \tau}=\epsilon P_{1}(a)+O(\epsilon), \\
& \frac{d \psi}{d \tau}=1+\epsilon Q_{1}(a)+O(\epsilon),
\end{aligned}
$$

and the functions $P_{1}(a)$ and $Q_{1}(a)$ are given by:

$$
\begin{gathered}
P_{1}(a)=-\frac{1}{2 \pi} \int_{0}^{2 \pi} f(a \cos \psi) \sin \psi d \psi, \\
Q_{1}(a)=-\frac{1}{2 \pi a} \int_{0}^{2 \pi} f(a \cos \psi) \cos \psi d \psi .
\end{gathered}
$$

On inserting the explicit expression (22) for $f(a \cos \psi)$ one gets:

$$
\begin{gathered}
P_{1}(a)=0 \\
Q_{1}(a)=-\frac{2 \beta \mathcal{G}}{\pi \sqrt{B_{1}^{2}+(\beta \mathcal{G} a)^{2}}} \int_{0}^{\pi / 2} \frac{\left(1-\sin ^{2} \psi\right)}{\sqrt{1-k^{2} \sin ^{2} \psi}} d \psi
\end{gathered}
$$

where

$$
k^{2}=\frac{(\beta \mathcal{G} a)^{2}}{B_{1}^{2}+(\beta \mathcal{G} a)^{2}} .
$$

Eq. (28) can be written as:

$$
Q_{1}(a)=-\frac{2 \beta \mathcal{G}}{\pi k^{2} \sqrt{B_{1}^{2}+(\beta \mathcal{G} a)^{2}}}\left[\left(k^{2}-1\right) K(k)+E(k)\right],
$$

where $K(k)$ and $E(k)$ are the complete elliptic integrals of the first and second kind. When $k \simeq 1$ elliptic integrals can be approximated by:

$$
\begin{gathered}
K(k) \approx \ln \frac{4}{\sqrt{1-k^{2}}}+\frac{1}{4}\left(\ln \frac{4}{\sqrt{1-k^{2}}}-\frac{1}{2}\right)\left(1-k^{2}\right) \\
E(k) \approx 1+\frac{1}{2}\left(\ln \frac{4}{\sqrt{1-k^{2}}}-\frac{1}{2}\right)\left(1-k^{2}\right)
\end{gathered}
$$

In the first order approximation the frequency shift is:

$$
\begin{aligned}
& \delta \omega_{c} \simeq \epsilon Q_{1}(a)=\frac{2}{\pi} \frac{\alpha \beta^{2} \mathcal{G}|\mathcal{G}|}{\sqrt{B_{1}^{2}+(\beta \mathcal{G} a)^{2}}}\{1+ \\
& \left.\frac{1}{2} \frac{B_{1}^{2}}{B_{1}^{2}+(\beta \mathcal{G} a)^{2}}\left[\ln \left(\frac{4 \sqrt{B_{1}^{2}+(\beta \mathcal{G} a)^{2}}}{B_{1}}\right)+\frac{1}{2}\right]\right\}
\end{aligned}
$$

In the approximation $a \approx A$, one obtains Eq. (17).
[1] B.C. Stipe, H.J. Mamin, C.S. Yannoni, T.D. Stowe, T.W. Kenny and D. Rugar, Phys. Rev. Lett. 87, 277602 (2001).

[2] D. Rugar, R. Budakian, H.J. Mamin and B.W. Chui, Nature 430, 329 (2004).

[3] G.P. Berman, D.I. Kamenev and V.I. Tsifrinovich, Phys. Rev. A 66, 023405 (2002).
[4] G.P. Berman, F. Borgonovi and V.I. Tsifrinovich, Quantum Information and Computation, 4 , 102 (2004).

[5] N.N. Bogolubov and Y.A. Mitropolsky, Asymptotic methods in the theory of non-linear oscillations, (translated from Russian), Delhi, Hindustan Pub. Corp. (1961). 